\title{
Reflux characteristics of 113 GERD patients with abnormal 24-h multichannel intraluminal impedance-pH tests
}

\author{
Yuan-yuan NIAN ${ }^{\dagger}$, Cheng FENG, Fu-chun JING, Xue-qin WANG, Jun ZHANG ${ }^{\dagger *}$ \\ (Division of Gastroenterology, the Second Affiliated Hospital of Xi' an Jiaotong University, Xi'an 710003, China) \\ "E-mail: nianyuanyuanneve@126.com; jun3z@163.com \\ Received Jan. 24, 2015; Revision accepted June 7, 2015; Crosschecked Aug. 10, 2015
}

\begin{abstract}
Objective: To analyze reflux parameters by means of combined multichannel intraluminal impedance and $\mathrm{pH}(\mathrm{MII}-\mathrm{pH})$ monitoring in patients with gastroesophageal reflux disease (GERD) symptoms off medication, and to find the reflux characteristics of Chinese GERD patients and the influences of gender, age, body posture, and body mass index (BMI) on gastroesophageal reflux (GER). Methods: Between Dec. 2008 and May 2014, 125 patients with typical GERD symptoms were subjected to 24-h MII-pH monitoring. Twelve patients with normal MII-pH profiles were not considered for analysis. The reflux parameters of 113 GERD patients with abnormal MII-pH results were analyzed. Results: (1) DeMeester scores were above the normal range in $46.90 \%(53 / 113)$ of GERD patients. Weakly acidic refluxes were prevalent in GERD patients, and the frequency of abnormal weakly acidic reflux was $75.22 \%(85 / 113)$. The frequencies of abnormal symptom index (SI) and symptom association probability (SAP) were $19.47 \%(22 / 113)$ and $14.16 \%$ (16/113), respectively. (2) The frequencies of DeMeester scores, the \%time at $\mathrm{pH}<4$, and the numbers of reflux episodes and of long reflux episodes $>5$ min were significantly higher in male patients than in female patients. (3) The \%time at $\mathrm{pH}<4$ was much higher during upright periods than during supine periods. During supine periods, $31.86 \%$ (36/113) of GERD patients had delayed bolus clearance time, compared with $19.47 \%$ (22/113) during upright periods. (4) The number of abnormal DeMeester scores, \%time at $\mathrm{pH}<4$, and the number of acid refluxes during upright periods were significantly higher in obese GERD patients than in GERD patients with a normal BMI. Overweight GERD patients also had many more acid refluxes during upright periods than GERD patients with a normal BMI. Conclusions: Weakly acidic refluxes were prevalent in Chinese GERD patients. The factors male, gender, upright position, obesity (BMI 225), but not age, may increase the frequency and severity of GER.
\end{abstract}

Key words: Gastroesophageal reflux disease (GERD), Multichannel intraluminal impedance-pH (MII-pH), Reflux characteristics, Off medication

doi: $10.1631 /$ jzus.B1500027

Document code: A

CLC number: R571

\section{Introduction}

Gastroesophageal reflux disease (GERD) is one of the most common digestive diseases, with a high prevalence and a variety of clinical symptoms and complications. The incidence of GERD has been increasing in China recently. At present, proton pump inhibitors (PPIs) are the first-line medication for the treatment of GERD. However, this treatment may not be suitable for some symptoms of GERD patients,

\footnotetext{
${ }^{\ddagger}$ Corresponding author

(iD ORCID: Yuan-yuan NIAN, http://orcid.org/0000-0002-0989-9417 (C) Zhejiang University and Springer-Verlag Berlin Heidelberg 2015
}

and it has uncertain effect in preventing Barrett's esophagus (BE), intestinal metaplasia (IM) and adenocarcinoma (American Gastroenterological Association, 2011; Fitzgerald et al., 2014). GERD patients with different symptoms may have different reflux profiles and should be given different treatments. For example, weakly acidic refluxes are more frequent than acid refluxes in patients with atypical symptoms of GERD, whereas acid refluxes still represent the most common phenomenon in GERD patients with typical symptoms (Grossi et al., 2013).

Combined multichannel intraluminal impedance and $\mathrm{pH}$ (MII-pH) monitoring is considered the most sensitive tool for gastroesophageal reflux (GER) 
assessment in GERD patients as it can detect all types of reflux (gas, liquid, acid, weakly acidic and weakly alkaline reflux).

Our aim was to analyze reflux parameters by means of MII-pH monitoring in patients with typical GERD symptoms off medication, to find the reflux characteristics of Chinese GERD patients, and to determine the influences of gender, age, body posture, and body mass index (BMI) on GER.

\section{Subjects and methods}

\subsection{Subjects}

Inclusion criteria: GERD patients with typical symptoms (heartburn and/or reflux) lasting for more than three months and age $\geq 20$ years. Patients were asked to discontinue any medication that would influence esophageal motor function or gastric fluid acidity at least seven days before performing MII-pH monitoring.

Exclusion criteria: history of thoracic, esophageal or gastric surgery, or underlying psychiatric illness.

\subsection{MII-pH monitoring}

MII-pH monitoring was performed using an MII-pH monitoring system (MMS, Enschede, the Netherlands), with a data logger and a combined impedance- $\mathrm{pH}$ catheter, which has one $\mathrm{pH}$ electrode and six impedance electrodes. After calibration, the impedance-pH catheter was inserted trans-nasally into the esophagus until the lower tip reached the gastric cavity. Then, the catheter was adopted and fixed, allowing monitoring of changes in intraluminal $\mathrm{pH}$ at 5 and impedance at 3, 5, 7, 9, 15, and $17 \mathrm{~cm}$ above the lower esophageal sphincter. Subjects were discharged and asked to maintain normal activities and sleep schedules, to eat their usual meals at their normal time, and to record symptoms, meal time, and posture changes. Impedance and $\mathrm{pH}$ data were collected on a compact flash card for $24 \mathrm{~h}$, then downloaded onto a computer and analyzed using a dedicated software program. The definitions of reflux episodes are shown in Table 1.

Table 1 Definitions of reflux episodes

\begin{tabular}{ll}
\hline \multicolumn{1}{c}{ Reflux episode } & \multicolumn{1}{c}{ Definition } \\
\hline Acid reflux & Reflux with nadir $\mathrm{pH}<4$ \\
Weakly acidic reflux & Reflux with nadir $4 \leq \mathrm{pH}<7$ \\
Weakly alkaline reflux & Reflux with nadir $\mathrm{pH} \geq 7$ \\
\hline
\end{tabular}

Twenty-four-hour MII-pH monitoring results were defined as abnormal when either the DeMeester score, acid reflux, weakly acidic reflux, weakly alkaline reflux, symptom index (SI), or symptom association probability (SAP) was above the upper normal limit. The normal values of the MMS MII-pH system were reported by Zerbib et al. (2005).

\subsection{Statistical analysis}

Data are presented as mean \pm standard error or median and interquartile range (M (IQR)). Differences between groups were compared using the Chi-square or Mann-Whitney test depending on the data, and considered to be significantly different when $P$ values were $<0.05$.

\section{Results}

\subsection{Demographic data}

Between Dec. 2008 and May 2014, 125 patients with typical GERD symptoms were subjected to 24-h MII-pH monitoring. Twelve patients with normal MII-pH profiles were not considered for analysis. Abnormal results were found in 90.4\% (113/125) of patients. The demographic data of the 113 GERD patients are shown in Table 2.

Table 2 Demographic data of the 113 GERD patients

\begin{tabular}{lc}
\hline \multicolumn{1}{c}{ Parameter } & Number \\
\hline Gender (male/female) & $48 / 65$ \\
Age distribution & \\
Group A (20-39 years) & 14 \\
Group B (40-49 years) & 31 \\
Group C (50-59 years) & 41 \\
Group D ( $\geq 60$ years) & 27 \\
BMI distribution & \\
Lean body mass $(\mathrm{BMI}<18.5)$ & 13 \\
Normal $(18.5 \leq \mathrm{BMI}<22.9)$ & 45 \\
Overweight $(23.0 \leq \mathrm{BMI}<24.9)$ & 24 \\
Obesity $(\mathrm{BMI} \geq 25.0)$ & 21 \\
\hline
\end{tabular}

The patients aged $(52.69 \pm 12.73)$ years (range $21-93$ years)

DeMeester scores were above the normal range in $46.90 \%(53 / 113)$ of GERD patients (Fig. 1). Weakly acidic refluxes were prevalent in GERD patients. The frequency of abnormal weakly acidic reflux was $75.22 \%(85 / 113)$, and frequencies of abnormal SI and SAP were $19.47 \%$ (22/113) and $14.16 \%$ (16/113), respectively. 




Fig. 1 Frequencies of abnormal results for reflux parameters in the 113 GERD patients

\subsection{Influences of gender and age on GER}

Patients were subgrouped according their genders. The frequencies of abnormal reflux parameters in male and female patients are reported in Fig. 2. The frequencies of abnormal DeMeester scores, \%time at $\mathrm{pH}<4$, number of reflux episodes, and number of long reflux episodes were significantly higher in male than in female patients $(58.33 \%$ vs. $38.46 \%, 56.25 \%$ vs. $38.46 \%, 33.33 \%$ vs. $13.85 \%$, and $50.00 \%$ vs. $26.15 \%$, respectively). The frequencies of abnormal acid refluxes, weakly acidic refluxes, and weakly alkaline refluxes were not significantly different between males and females.

The 113 GERD patients were subdivided into four groups according to their ages: Group A (2039 years), Group B (40-49 years), Group C (5059 years), and Group D ( $\geq 60$ years). There were no significant differences among the four age groups for reflux parameters.

\subsection{Influence of body posture on GER}

The \%time at $\mathrm{pH}<4$ was much higher during upright periods than during supine periods (Table 3 ). During supine periods, $31.86 \%$ (36/113) of GERD patients had delayed bolus clearance time, compared with $19.47 \%$ (22/113) during upright periods. Other reflux parameters showed no statistically significant differences in relation to body posture.

\subsection{Influence of BMI on GER}

Among 113 GERD patients, 103 patients had BMI information, and they were subgrouped into four groups according to their BMI values: 13 patients with lean body mass $(\mathrm{BMI}<18.5), 45$ patients with normal BMI $(18.5 \leq \mathrm{BMI}<22.9), 24$ patients with overweight $(23.0 \leq \mathrm{BMI}<24.9)$, and 21 obese patients

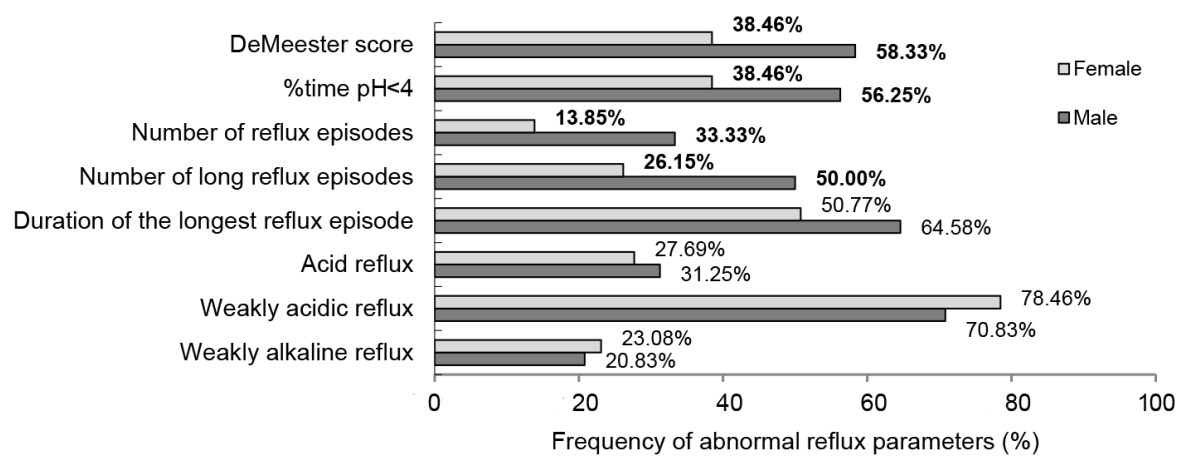

Fig. 2 Frequencies of abnormal reflux parameters in male and female GERD patients Frequencies associated with significant differences $(P<0.05)$ are shown in bold

Table 3 Numbers or frequencies of abnormal reflux parameters during upright and supine periods in GERD patients

\begin{tabular}{llcccc}
\hline \multirow{2}{*}{ Period } & \multirow{2}{*}{ \%time at $\mathrm{pH}<4^{*}$} & \multicolumn{4}{c}{ Frequency of abnormal reflux parameters (\%) } \\
\cline { 3 - 5 } & & Acid reflux & Weakly acidic reflux & Weakly alkaline reflux & Bolus clearance time $^{*}$ \\
\hline Upright & $3.8(0.8,13.05)$ & 14.16 & 56.64 & 20.35 & 19.47 \\
Supine & $1.5(0,11.4)$ & 22.12 & 64.60 & 10.62 & 31.86 \\
\hline
\end{tabular}

Data for \%time at $\mathrm{pH}<4$ are presented as $\mathrm{M}(\mathrm{IQR})(n=113)$, data of other parameters are presented as percentages of abnormal data. ${ }^{*} P<0.05$, significant difference between upright and supine periods in GERD patients 
Table 4 Incidence of BMI on reflux parameters in GERD patients

\begin{tabular}{|c|c|c|c|c|c|c|c|c|}
\hline \multirow{2}{*}{ Group } & \multirow{2}{*}{$\begin{array}{l}\text { DeMeester } \\
\text { score }\end{array}$} & \multirow{2}{*}{$\begin{array}{c}\text { \%time at } \\
\mathrm{pH}<4\end{array}$} & \multicolumn{2}{|c|}{ Acid reflux } & \multicolumn{2}{|c|}{ Weakly acidic reflux } & \multicolumn{2}{|c|}{ Weakly alkaline reflux } \\
\hline & & & Up & $\mathrm{Su}$ & Up & $\mathrm{Su}$ & Up & $\mathrm{Su}$ \\
\hline $\begin{array}{l}\text { Lean body mass } \\
(n=13)\end{array}$ & $\begin{array}{c}11.25 \\
(8.68,62.17)\end{array}$ & $\begin{array}{c}2.80 \\
(2.10,20.50)\end{array}$ & $\begin{array}{c}3.20 \\
(0,18.15)\end{array}$ & $\begin{array}{c}1.00 \\
(0,9.85)\end{array}$ & $\begin{array}{c}12.20 \\
(3.05,45.85)\end{array}$ & $\begin{array}{c}2.10 \\
(1.00,7.80)\end{array}$ & $\begin{array}{c}0 \\
(0,1.55)\end{array}$ & $\begin{array}{c}0 \\
(0,0)\end{array}$ \\
\hline $\begin{array}{c}\text { Normal } \\
(n=45)\end{array}$ & $\begin{array}{c}6.66 \\
(1.44,34.93)\end{array}$ & $\begin{array}{c}1.65 \\
(0.30,10.15)\end{array}$ & $\begin{array}{c}5.80 \\
(1.85,18.23)\end{array}$ & $\begin{array}{c}1.00 \\
(0,3.20)\end{array}$ & $\begin{array}{c}17.55 \\
(3.95,55.23)\end{array}$ & $\begin{array}{c}4.50 \\
(0.75,12.15)\end{array}$ & $\begin{array}{c}1.00 \\
(0,4.33)\end{array}$ & $\begin{array}{c}0 \\
(0,0)\end{array}$ \\
\hline $\begin{array}{l}\text { Overweight } \\
(n=24)\end{array}$ & $\begin{array}{c}14.32 \\
(3.37,35.77)\end{array}$ & $\begin{array}{c}4.15 \\
(0.83,12.33)\end{array}$ & $\begin{array}{c}14.70 \\
(2.38,32.98)^{*}\end{array}$ & $\begin{array}{c}2.10 \\
(0,3.18)\end{array}$ & $\begin{array}{c}19.50 \\
(8.58,77.45)\end{array}$ & $\begin{array}{c}5.25 \\
(1.68,19.33)\end{array}$ & $\begin{array}{c}1.05 \\
(0,2.18)\end{array}$ & $\begin{array}{c}0 \\
(0,0.75)\end{array}$ \\
\hline $\begin{array}{l}\text { Obese } \\
(n=21)\end{array}$ & $\begin{array}{c}50.57 \\
(4.24,83.24)^{*}\end{array}$ & $\begin{array}{c}11.50 \\
(1.10,26.25)^{*}\end{array}$ & $\begin{array}{c}10.60 \\
(4.65,26.05)^{*}\end{array}$ & $\begin{array}{c}2.10 \\
(0.50,4.75) \\
\end{array}$ & $\begin{array}{c}25.60 \\
(12.05,60.75) \\
\end{array}$ & $\begin{array}{c}2.10 \\
(1.10,12.05) \\
\end{array}$ & $\begin{array}{c}0 \\
(0,4.75) \\
\end{array}$ & $\begin{array}{c}0 \\
(0,0) \\
\end{array}$ \\
\hline
\end{tabular}

Data are presented as M (IQR). Up: during upright periods; Su: during supine periods. ${ }^{*} P<0.05$ compared with the normal control

$(\mathrm{BMI} \geq 25.0)$. The numbers of DeMeester scores, $\%$ time at $\mathrm{pH}<4$, and acid refluxes during upright periods were significantly higher in obese GERD patients than in those with a normal BMI (50.57 (4.24, $83.24)$ vs. $6.66(1.44,34.93), 11.50(1.10,26.25)$ vs. $1.65(0.30,10.15)$, and $10.60(4.65,26.05)$ vs. 5.80 $(1.85,18.23)$, respectively, as shown in Table 4). In addition, overweight GERD patients also had much more acid refluxes during upright periods than GERD patients with normal BMI $(14.70(2.38,32.98)$ vs. $5.80(1.85,18.23))$.

\section{Discussion}

GERD is a digestive disease with a variety of troublesome reflux symptoms and/or complications, such as reflux esophagitis (RE), BE, and adenocarcinoma. The incidence of GERD has been increasing recently, in relation to the financial stress and poor quality of life of patients (Vakil, 2010). In Asian countries, the prevalence of GERD has been increasing because of changing dietary patterns, obesity, and other factors.

Several procedures are used to identify GER. The most widely used clinical procedures include: GERD-questionnaire (GERD-Q), endoscopy, pH monitoring, MII-pH monitoring, esophageal manometry, and the PPI test. Among them, MII-pH monitoring is currently considered the most sensitive tool for GER detection in GERD patients.

Wang et al. (2011) and Frazzoni et al. (2009) reported that combined MII-pH monitoring increased overall diagnostic yields. In this study, we evaluated reflux parameters by 24-h MII-pH monitoring in GERD patients off medication. Of 113 GERD patients confirmed by MII-pH monitoring, only $46.90 \%$ had abnormal DeMeester scores. Schindlbeck et al.
(1987) reported that the DeMeester score was not necessarily superior to the \%time at $\mathrm{pH}<4$ in discriminating GER. Our study confirmed this opinion: the $\%$ time at $\mathrm{pH}<4$ and DeMeester scores were above the normal range in $46.02 \%$ and $46.90 \%$ of GERD patients, respectively, and there was no significant difference between these two parameters in discriminating GER. Frequencies of abnormal SI and SAP were very low in this study. The reason may be that although patients complained about troublesome symptoms in their daily life, they may not have had these symptoms when undergoing MII-pH monitoring.

Guidelines recommend PPIs as the first line of treatment for GERD patients (Katz et al., 2013), but a considerable proportion of patients does not respond to PPIs (Clayton et al., 2012; Vela, 2014). Clayton et al. (2012) showed that PPI treatment can transform acid refluxes into weakly acidic refluxes, but can not decrease the total number of reflux events. In this study, weakly acidic refluxes were prevalent in GERD patients off medication. The number of weakly acidic refluxes was above the normal range in $75.22 \%$ of GERD patients, whereas the frequencies of abnormal acid refluxes and weakly alkaline refluxes were $28.32 \%$ and $22.12 \%$, respectively. The finding of weakly acidic reflux as the major type of reflux was different from the results of previous studies (Xiao et al., 2009). The reason is unclear. In a weakly acidic environment bile reflux can impair esophageal mucosal integrity and provoke dilated intercellular spaces (Farre et al., 2008). With MII-pH monitoring, it has been established that weakly acidic reflux also plays a role in the pathogenesis and natural history of GERD. Weakly acidic refluxes are more frequent than acid refluxes in patients with atypical symptoms of GERD (Grossi et al., 2013). Weakly acidic refluxes may be more important for BE and non-erosive reflux disease (NERD) patients than separate acid 
reflux (Wang et al., 2011), and are the major cause of persistent symptoms in patients with NERD, who are being treated with PPIs (Hiroshi et al., 2012). Studies of weakly acidic reflux and BE showed that weakly acidic refluxes were more prevalent in long-segment $\mathrm{BE}$ and short-segment $\mathrm{BE}$ patients than in $\mathrm{RE}$ and normal volunteers. Weakly acidic reflux may play a role in the pathogenesis of BE (Gutschow et al., 2008) and persistent intestinal metaplasia after ablation in patients with BE (Krishnan et al., 2012).

In our study, the frequencies of abnormal DeMeester scores, \%time at $\mathrm{pH}<4$, and numbers of reflux episodes were much higher in male than in female GERD patients. This means that the frequency and severity of symptoms were higher in male than in female patients. Although the incidence of GERD in the male and female populations was similar, the incidence of complications, such as RE, BE, and adenocarcinoma, was much higher in the male than in female population.

Aging is one of the risk factors for GERD and its complications (El-Serag et al., 2009; Fitzgerald et al., 2014). When GERD patients were subdivided into four groups according their ages, there were no significant differences among the four groups for reflux parameters. We confirmed that the severity of symptoms was not higher in older than in younger GERD patients. Age was not directly associated with GERD and its complications. Older patients were inclined to have a longer duration of GERD, and that may be a risk factor for GERD complications.

Guidelines for the treatment of GERD recommend raising the head of the bed to reduce symptoms in GERD patients with nocturnal reflux. This implies that a supine position could aggravate reflux. However, Frazzoni et al. (2009) reported that refluxes were more prevalent when patients were in an upright position than when they were in a supine position. We compared the reflux parameters during supine and upright periods. Although bolus clearance time was delayed during supine periods, the \%time at $\mathrm{pH}<4$ was much higher during upright than during supine periods. The reasons for this phenomenon are still unclear, but may be related to meals or activities during upright periods.

Obese GERD patients had higher DeMeester scores and \%time at $\mathrm{pH}<4$, and more acid refluxes than patients with a normal BMI. This confirmed that a BMI of $\geq 25$ was related to GER in Chinese GERD patients, especially those with acid refluxes.

The main limitation of this study was that it was retrospective. This may have reduced the accuracy of the information. Also, we could not obtain endoscopic and manometric information. However, since all patients included in our study had reflux symptoms and abnormal refluxes confirmed by MII-pH, we are sure that we enrolled only GERD patients.

In conclusion, weakly acidic refluxes were prevalent in Chinese GERD patients; the factors male gender, upright position, obesity (BMI $\geq 25)$, but not age, may increase the frequency and severity of GER.

\section{Compliance with ethics guidelines}

Yuan-yuan NIAN, Cheng FENG, Fu-chun JING, Xue-qin WANG, and Jun ZHANG declare that they have no conflict of interest.

All procedures followed were in accordance with the ethical standards of the responsible committee on human experimentation (institutional and national) and with the Helsinki Declaration of 1975, as revised in 2008 (5). Informed consent was obtained from all patients for being included in the study.

\section{References}

American Gastroenterological Association, 2011. American Gastroenterological Association medical position statement on the management of Barrett's esophagus. Gastroenterology, 140(3):1084-1091. [doi:10.1053/j.gastro. 2011.01.030]

Clayton, S.B., Rife, C.C., Singh, E.R., et al., 2012. Twice-daily proton pump inhibitor therapy dose not decrease the frequency of reflux episodes during nocturnal recumbency in patients with refractory GERD: analysis of 200 patients using multichannel intraluminal impedance-pH testing. Dis. Esophagus, 25(8):682-686. [doi:10.1111/j. 1442-2050.2011.01310.x]

El-Serag, H., Hill, C., Jones, R., 2009. Systematic review: the epidemiology of gastroesophageal reflux disease in primary care, using the UK general practice database. Aliment. Pharm. Ther., 29(5):470-480. [doi:10.1111/j. 1365-2036.2008.03901.x]

Farre, R., van Malenstein, H., de Vos, R., et al., 2008. Short exposure of oesophageal mucosa to bile acid, both in acidic and weakly acidic conditions, can impair mucosal integrity and provoke dilated intercellular spaces. Gut, 57(10):1366-1374. [doi:10.1136/gut.2007.141804]

Fitzgerald, R.C., Pietro, M.D., Ragunath, K., et al., 2014. British Society of Gastroenterology guidelines on the diagnosis and management of Barrett's oesophagus. Gut, 63(1):7-42. [doi:10.1136/gutjnl-2013-305372]

Frazzoni, M., Savarino, E., Manno, M., et al., 2009. Reflux 
patterns in patients with short-segment Barrett's oesophagus: a study using impedance-pH monitoring off and on proton pump inhibitor therapy. Aliment. Pharmacol. Ther., 30(5):508-515. [doi:10.1111/j.1365-2036.2009. 04063.x]

Grossi, L., di Tullio, A.M., Pagliaro, M., et al., 2013. Role of weakly acidic refluxes in the atypical symptoms of GERD A pH-imedance study performed off-PPI therapy. Digest. Liver Dis., 45(2):S111. [doi:10.1016/S1590-8658(13) 60307-2]

Gutschow, C., Bludau, M., Vallböhmer, D., et al., 2008. NERD, GERD, and Barrett's esophagus: role of acid and non-acid reflux revisited with combined $\mathrm{pH}$-impedance monitoring. Digest. Dis. Sci., 53(12):3076-3081. [doi:10. 1007/s10620-008-0270-6]

Hiroshi, Y., Kiyoshi, A., Takumi, F., et al., 2012. Combined pH-impedance monitoring and high-resolution manometry of Japanese patients treated with proton-pump inhibitors for persistent symptoms of non-erosive reflux disease. $J$. Smooth Muscle Res., 48(5):125-135. [doi:10.1540/jsmr. 48.125]

Katz, P.O., Gerson, L.B., Vela, M.F., 2013. American college of gastroenterology. Guidelines for the diagnosis and management of gastroesophageal reflux disease. Am. $J$. Gastroenterol., 108(3):308-328. [doi:10.1038/ajg.2012. 444]

Krishnan, K., Pandolfino, J.E., Kahrilas, P.J., et al., 2012. Increased risk for persistent intestinal metaplasia in patients with Barrett's esophagus and uncontrolled reflux before radiofrequency ablation. Gastroenterology, 143(3): 576-581. [doi:10.1053/j.gastro.2012.05.005]

Schindlbeck, N.E., Heinrich, C., Konig, A., et al., 1987. Optimal thresholds, sensitivity, and specificity of long-term $\mathrm{pH}$-metry for the detection of gastroesophageal reflux disease. Gastroenterology, 93(1):85-90.

Vakil, N., 2010. Disease definition, clinical manifestations, epidemiology and natural history of GERD. Best Pract. Res. Clin. Gastroenterol., 24(6):759-764. [doi:10.1016/j. bpg.2010.09.009]

Vela, M.F., 2014. Medical treatments of GERD: the old and new. Gastroenterol. Clin. North Am., 43(1):121-133. [doi:10.1016/j.gtc.2013.12.001]

Wang, K., Duan, L.P., Ge, Y., et al., 2011. Diagnostic values of GerdQ, 24-h ambulatory oesophageal $\mathrm{pH}$ and impedance-pH monitoring in Barrett's esophagus, reflux esophagitis and non-erosive reflux disease. Nat. Med. J. China, 91(18):1228-1232 (in Chinese). [doi:10.3760/cma. j.issn.0376-2491.2011.18.002]

Xiao, Y.L., Lin, J.K., Cheung, T.K., et al., 2009. Reflux profile of Chinese gastroesophageal reflux disease patients with combined multichannel intraluminal impedance-pH monitoring. J. Gastroenterol. Hepatol., 24(6):1113-1118. [doi:10.1111/j.1440-1746.2009.05861.x]

Zerbib, F., Varannes, S.B., Roman, S., et al., 2005. Normal values and day-to-day variability of 24-h ambulatory oesophageal impedance-pH monitoring in a BelgianFrench cohort of healthy subjects. Aliment. Pharmacol. Ther., 22(10):1011-1021. [doi:10.1111/j.1365-2036.2005. 02677.x]

\section{中文概要}

题 目: 24 小时食管多通道腔内阻抗-pH 监测阳性的 113 例胃食管反流病（GERD）患者的胃食管反流 特点

目 的：通过分析多通道腔内阻抗-pH 监测（MII-pH）阳 性的胃食管反流病（GERD）患者的各项反流指 标, 探讨我国 GERD 患者的胃食管反流特点, 以 及性别、年龄、体位和体质指数 (BMI) 对反流 的影响。

创新点: 入组患者均经 MII-pH 监测证实具有客观存在的 胃食管反流, 分析体位和 BMI 对反流的影响, 并 且发现我国 GERD 患者普遍存在弱酸反流。

方 法: 有典型反流症状的疑诊 GERD 患者, 进行 $24 \mathrm{~h}$ MII-pH 监测, 对监测结果阳性的患者纳入研究对 象, 分析其各反流指标以及性别、年龄、体位和 BMI 对反流的影响。

结 论: 我国 GERD 患者普遍存在弱酸反流, 男性、直立 体位、肥胖 (BMI 225$)$ 可促进胃食管反流的发生, 且年龄不是胃食管反流的危险因素。

关键词: 胃食管反流病 (GERD) ; 多通道腔内阻抗-pH 监测（MII-pH）；反流特点 